\title{
Socio-Economic Interaction - Addressing Problems of Safety in the Community
}

\author{
Tverdynin N.M. \\ The Civil Defence Academy of EMERCOM of Russia, \\ Khimki, Russia, \\ tvernick@mail.ru, alishari@yandex.ru
}

Grishakina N.I.

\author{
Yaroslav-the-Wise Novgorod State University, \\ Veliky Novgorod, Russia, \\ gnisemm@mail.ru
}

\author{
Tikhonova O.A. \\ Russian State Research Center - \\ Burnasyan Federal Medical Biophysical Center \\ of Federal Medical Biological Agency, \\ Moscow, Russia, \\ ttx_2001@mail.ru
}

\author{
Sharifullina L.R. \\ The Civil Defence Academy of EMERCOM of Russia, \\ Khimki, Russia, \\ alishari@yandex.ru
}

\begin{abstract}
Modern society is fundamentally different from all human communities that existed during the development of world civilization. The world has become a technosociety with a number of characteristics that cannot be correlated with the formations existed before. We can talk about an established society, where scientific and technological progress is not only a guarantee of development, but also a condition for existence, which is very noticeable in such areas as healthcare, energy industry, and the production of various materials. Unlike all previous civilizations, this society has a number of separate social segments that are not only interconnected, but also interdependent. Such a structure, which has no analogues in the past, requires a new approach to education, which should simultaneously be both highly-specialized and interdisciplinary, while performing both formative and educational functions. The safety of the modern world largely depends on the correct balance of educational principles, on the quality of education, as in the case of manmade disasters (Chernobyl and Fukushima) and the SARS-CoV-2 (COVID-19) viral pandemic in 2020. These events became agents for new methods of assessing the various factors mutual influence on such phenomena. At the same time, there is an interest in creating private methods that allow predicting the possible effectiveness of various economic and other measures to reduce the risk of emergencies and improve safety.
\end{abstract}

Keywords-education, safety, forecasting, model, technosphere, COVID-19

\section{INTRODUCTION}

When attempting to comprehensively study the problems that affect the safety of society from a variety of threats and challenges associated with its globalization and technogenicity, it is not enough to have data only on individual segments that make up the problem [1,2]. Of particular importance is accurate forecasting for such an area as medicine, where a significant amount of economic investment is interconnected with the maximum social effect.

The concept of patient care must be balanced with the safety of health care, the patient himself and society. This is achievable through a combination of technical means and administrative controls [3].

At the same time, it is necessary to take into account a large number of completely heterogeneous factors that are derived from various independent segments: medical activities themselves (including personnel training at all levels), technical and technological equipment, economic opportunities and qualitatively other segments related to the level of sociopolitical development of society and socio-psychological characteristics of various population groups.

\section{PROBlem STATEMENT}

Taking into account the abovementioned, there was made an attempt to demonstrate the need to create models accommodating the heterogeneity of factors and form the basis for forecasting further actions, including economic characteristics, using the example of a classical medical study on solving specific issues that study the principles of infection prevention, the infection caused by SARS-CoV-2 in the staff of a "COVID-19" hospital. A similar problem was solved by researchers from Mount Sinai Health System, New York [4].

It was assumed that the medical personnel training requires not only increased funding, but also the consideration of factors that are not directly related to medical problems.

\section{RESEARCH QUESTIONS}

3.1 Comparison of different categories of health workers who have been infected in the course of the patients infected with SARS-CoV-2 (COVID-19) treatment. 
3.2 Comparison of different categories of health workers, depending on their professional training in the field of infectious diseases.

3.3 Analysis of the impact of various factors related to different segments (economic, technological, social and others) on the development of the safety - education relationship.

3.4 Determination of ways to build a model that reflects the interaction of the above factors in order to build models that take into account both formalized and qualitative approaches to forecast the need for economic investments in the field of medical education and enlightenment.

\section{PURPOSE OF THE STUDY}

The purpose of the study is to identify approaches to building a model that allows us to take into account the influence of qualitatively different factors, including economic and psychological, on the training process of medical personnel at different levels. The analysis of the modular principle can give a significant advance in the issues of both the education of health workers and the education of the population on issues related to the risk of infectious diseases.

\section{RESEARCH METHODS}

The study on the effectiveness of complex preventive measures, including the use of drug preventive therapy, was conducted on the basis of the Russian State Research Center Burnasyan Federal Medical Biophysical Center of Federal Medical Biological Agency (RSRC - Burnasyan FMBC of FMBA) from April, 6 to July, 20, 2020. At the time of the organization of the infectious diseases department in a multidisciplinary hospital, 223 people were involved in providing care to patients infected with SARS-CoV2 (health workers, drivers, disinfectors, cooks, workers, etc.). Part of the team worked in conditions of increased risk of occupational infection and had indications for drug-specific prevention of a new coronavirus infection with a drug from the quinoline group. This observation group consisted of 48 men and 118 women aged $\geq 18$ and $\leq 69$ years, with an average age of $48.7 \pm 1.8$ years. Of these, 57 doctors of various profiles and 109 nurses. None of the study participants had previously worked in the infectious diseases department and did not have special knowledge of virology. Before starting work in the "red zone", all employees in a short time under the same type of program were trained in the use of personal protective equipment; they were informed about the ways of transmission and clinical manifestations of the virus. In all individuals of this group, the result of polymerase chain reaction (PCR) on SARS-Cov-2 smear from the oropharynx at the time of starting work in the infectious department was negative.

Participants in the study, which was used to help patients with COVID-19, were asked to fill out questionnaires. The basic questionnaire included questions related to job responsibilities, emotional state, physical fatigue, side effects from personal protective equipment (PPE) and preventive medication. The survey was conducted remotely via Messenger. In the course of the study, content analysis methods were used in combination with separate statistical methods. The effectiveness of the preventive measures was evaluated by such parameters as: the presence of the disease (confirmed by a PCR test or serological examination) during the work in the "red zone", the severity of the disease, the presence of pneumonia, confirmed by computer tomography.

\section{FINDINGS}

The analysis of the relationship of the quality of training, level of medical knowledge, the effect of preventive medication for the prevention of morbidity of personnel RSRC - Burnasyan FMBC of FMBA during the period of the "COVID hospital".

A total of $26(15.6 \%)$ cases of acute occupational disease COVID-19, confirmed by a test (PCR) or serological test, were recorded during the work of the infectious diseases department. In the group of persons injured in the discharge of their functions, there were 10 nurses, including those working in the anesthesiology department, 9 nurses and 3 cleaners (all women).

Doctors were less exposed to occupational infection, the disease was observed in 4 people ( 2 men, 2 women). The average age of all patients was $39.63 \pm 1.2$ years. All cases of occupational infection were recorded during the first two weeks after the opening of the infectious diseases department, during the period of mastering new skills and gaining experience with this type of infection.

Summary data on the number of patients of different occupational groups are presented in Fig. 1.

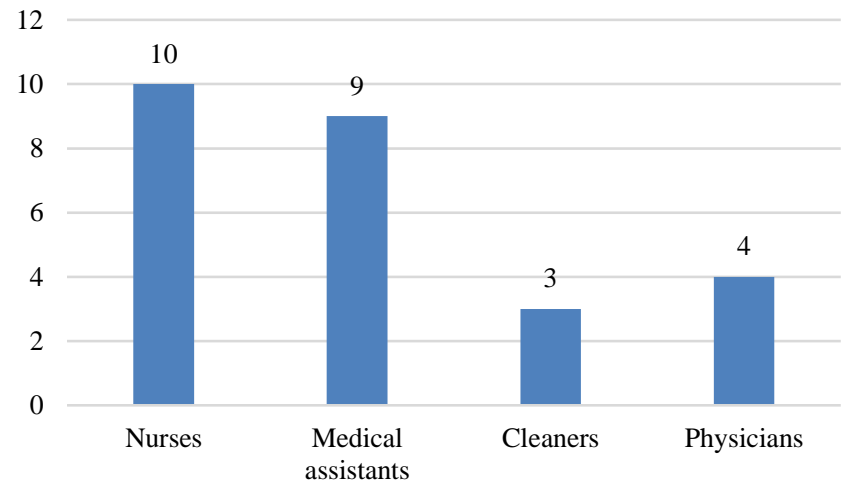

Fig. 1. Distribution of personnel infected with SARS-CoV-2 in discharge of their functions

We explain this distribution of patients among medical personnel by the level of education, the range of job responsibilities and age. Secondary and junior medical personnel, in accordance with their official duties, unlike doctors, had more frequent and prolonged contact with COVID19 patients and their biological material. This is evidenced by the data of other researchers [5].

The results of our work demonstrate that the severity of the course of pneumonia caused by COVID-19 depended, among other things, on the amount of non-specific drug prevention, as well as on age and position.

Thus, in the subgroup of employees who took medication for the prevention of COVID-19 with a full course (7 people), cases of severe pneumonia were not encountered, the duration of treatment in the hospital was on average 9.6 days. In persons who took a preventive medication once or an incomplete course (19 people) due to the development of undesirable side reactions, the disease was more severe, so moderate pneumonia was observed in 12 people, severe in three, and the average duration of hospitalization was 21.6 days. 
We believe that taking a drug in preventive doses does not exclude the possibility of infection with SARS-CoV-2, but against its background, there is an earlier recovery and smoothness of clinical symptoms, probably due to the antiinflammatory properties of the drug. The results obtained correspond to the data of the literature sources $[6,7]$. Thus, the risk group for occupational infection was dominated by middleaged women from among secondary and junior medical personnel with an appropriate level of education and, probably, the level of perception of new information associated with education.

Although our findings are not supported by statistically significant calculations due to the small sample size of patients, the information obtained fully confirms the need for more serious preliminary training of health workers at all levels involved in the fight against the pandemic. Since, in such cases, non-economic principles apply, and the results of recovery and recovery of working capacity are of the greatest importance, the need to develop new model approaches to the training process of personnel at all levels as a prevention of a pandemic like SARS-CoV-2 (COVID-19) is certainly necessary.

Returning to the analysis of the impact of various factors related to different segments and influencing the relationship between security and education, I would like to emphasize the following. Factors related to professional activity, namely: the level of personnel training, the qualifications of their individual categories, time (experience) of working in conditions of certain professional risks, can be quantified and relatively easily formalized [8]. Similarly, economic and technological factors can be quantified. Economic factors relate to the cost of staff salaries, including various kinds of qualification allowances and payments related to dangerous working conditions (as in the case of personnel who worked with SARS-CoV-2 (COVID-19).

The proposed scheme for analyzing the interaction of factors is shown in Fig. 2.

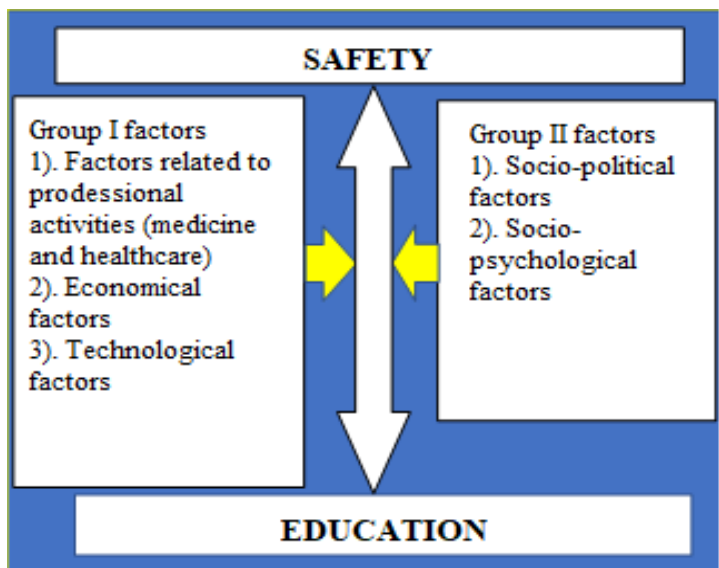

Fig. 2. Diagram of mutual interference of various factors on safety education

Technological factors include the capabilities of the equipment, including devices, tools, consumables and basic equipment of the hospital where the treatment is performed.

The factors of group II depend on the parameter, the formalization of which, of course, is possible, but when compared to the factors of the first group is much more difficult and can lead to erroneous conclusions. This is due to the fact that people's assessment of certain dangers is related both to their education, social affiliation and many other parameters, including national traditions, place of residence, etc.

Naturally, the current public mood can significantly affect the social order in the field of application of certain technologies related to treatment and investment in the production of medicines and equipment and the development of new models (economic segment of group I).

In order to draw a prognostic conclusion on the development of the situation with the real state of education related to safety, both medical and any other, it seems advisable not to try to formalize the factors of group II, but to leave them in the state of evaluation according to the "three-point system": "positive", "negative" and "the majority has not decided on the assessment". This is why this work differs significantly from a number of sources that develop model principles for solving various complex (multi-factor) problems $[9,10]$

If all the indicators of the group I segment show an upward trend, and both segments reflecting the factors of group II are positive, then the forecast for the development of the situation in the near future can be considered as completely optimistic. Accordingly, any negative dynamics in the indicators of factors in both groups reduces the positivity of the forecast. There may be a situation when there is a negative assessment of the danger factor in the society or in the government circles of a particular state. For example, in Sweden and a number of other states, the risk associated with SARS-CoV-2 (COVID-19) is generally sharply negative (albeit for a relatively short period. In this case, all potential positive dynamics in the segments of the first group will be inhibited.

\section{FINDINGS}

All cases of occupational infection with SARS-CoV-2 were recorded in the first month of the infectious hospital operation, when the experience of employees in the new conditions was insufficient. During this period of time, medical staff experienced fear for their health and the health of family members, anxiety about the side effects of PPE and drug prevention. This is evidenced by other authors of similar studies [11].

\section{CONCLUSION}

A comparison of different categories of health workers confirmed the intuitive assumption that the higher the employee's qualifications and educational level, the lower the probability of infection when working in the context of combating the SARS-CoV-2 (COVID-19) pandemic, other things being equal. To plan preventive measures for personnel training and preventive work among the population, taking into account social, psychological and other characteristics, it is necessary to consider various by their nature factors. When constructing appropriate models, it is proposed to abandon the absolute formalization of different nature indicators, for example, economic and socio-psychological.

This approach allows for a more accurate prediction process, since the parameters of each of the segments shown in Fig. 2 are not independent, but multifunctional. For example, economic factors, although they can be estimated relatively simply, since modern methods of economic analysis allow us to build such particular models and present them in a formalized form, they have a significant number of variables that are largely based on factors from other segments (for example, politics, etc.), which significantly increases the uncertainty of 
the forecast. In other words, it turns out that, although the result of such modeling is formally logical, its real value may be quite far from that obtained with the help of information and communication technologies (ICT). This is because in a probabilistic forecast, you should not talk about the exact value or even about a simple probability interval. In the interval obtained during forecasting, it is necessary to allocate zones of greater or lesser probability of the result. If you do not do this, there may be conceptual errors. We will give further examples of similar errors in other branches of knowledge to explain this idea.

For example, when considering the criteria for the possibility of a chemical reaction, well-known equations of physical chemistry are used, which will determine the flow of the process in one direction or another or the conditions of equilibrium, which to a certain extent can be considered as an analogy to the processes described by the first three groups of factors (Fig. 2). However, the equilibrium position in real physical and chemical systems, depending on a number of reasons, is not adequate to the calculated value, but lies in a certain range (and this must be taken into account by any specialist, unlike a student who solves such a problem as a training task).

If we talk about the second group of factors that operate most often with survey data, then it is appropriate to give an example with the ratings of politicians compiled by some parameters, for example, the frequency of mention in the media. It is well known that many politicians who have fairly high ratings will almost never have a chance as candidates for certain political posts. This analogy may also be false when applied to the problems discussed in the article.

As already shown, both groups of factors are not only heterogeneous, but also represent fundamentally different segments. Therefore, an attempt to build a model that takes into account such a diversity of factors, it seems, can be considered as quite promising.

The authors hope to offer examples of more advanced models of this kind related to the problems of not only medical, but also technosphere safety in the next article.

\section{References}

[1] O.D. Shipunova, and A.V. Deniskov, "Subjective Interactions under Conditions of Modern Technological Way", International Research Journal, 2018, vol. 1(67-4), pp. 136-140. (In Russ.). DOI: https://doi.org/10.23670/IRJ.2018.67.135

[2] G.B. Kleiner, M.A. Rybachuk, and D.V. Ushakov, "Psychological Factors of Economic Behavior: A Systemic View", Terra Economicus, 2018, vol. 16(1), pp. 20-35. (In Russ.). DOI: 10.23683/2073-6606-2018-16-120-36

[3] M.N. Midhun, R. Chandni, and P. Indira, "COVID-19: Striking a Balance between Health Care Provider Safety and Patient Care in the Indian Hospital Settings", J Assoc Physicians India, 2020, vol. 68(6), pp. 41-47.

[4] J. Ripp, L. Peccoralo, and D. Charney, "Attending to the Emotional WellBeing of the Health Care Workforce in a New York City Health System During the COVID-19 Pandemic", Academic Medicine, 2020, vol. 95(8), pp. 1136-1139. DOI: 10.1097/ACM.0000000000003414

[5] N.P. Mayr, S. Sernetz, F. Heitzer, M. Joner, and P. Tassani-Prell, "Occupational safety in the treatment of COVID-19 patients: Relevant laws and regulations for the treating personnel", Anaesthesist, 2020, vol. 69(10), pp. 712-716. DOI: 10.1007/s00101-020-00828-0

[6] F. Touret, and X. Lamballerie, "Of chloroquine and COVID-19", Antiviral Research, 2020, vol. 177. DOI: https://doi.org/10/1016/j.antiviral.2020.104762

[7] A.S. Kolbin, "An early assessment of the efficacy of medicines in the treatment of patients with COVID-19", Infection and Immunity, 2020, vol. 10(2), pp. 277-286. (In Russ.). DOI: https://doi.org/10.15789/22207619-AEA-1458

[8] M. Denning, E.T. Goh, A. Scott, G. Martin, S. Markar, K. Flott, S. Mason, J. Przybylowicz, M. Almonte, J. Clarke, J. Winter-Beatty, S. Chidambaram, S. Yalamanchili, B. Yong-Qiang Tan, A. Kanneganti, V. Sounderajah, M. Wells, S. Purkayastha, and J. Kinross, "What has been the impact of Covid-19 on Safety Culture? A case study from a large metropolitan teching hospital", Intenational Jounal of Environmental Research and Public Health, 2020, vol. 17(19), 7034. DOI: https://doi.org/10.1101/2020.06.15.20129080

[9] P.N. Vishnevskiy, "Method of Case Modeling", Higher Education in Russia, 2017, vol. 12, pp. 71-77. (In Russ).

[10] V.I. Gushchin, A.G. Vinokhodova, D.V. Kommissarova, M.S. Belakovsky, and O.I. Orlov, "Experiments with isolation: the past, present, and future", Aerospace and Environmental Medicine, 2018, vol. 52(4), pp. 5-16. (In Russ.).

[11] A. Tabah, M. Ramanan, K.B. Laupland, N. Buetti, A. Cortegiani, J. Mellinghoff, A.C. Morris, L. Camporota, N. Zappella, M. Elhadi, P. Povoa, K. Amrein, G. Vidal, L. Derde, M. Bassetti, G. Francois, N. Ssi Yan Kai, and J.J. De Waele, "Personal protective equipment and intensive care unit healthcare worker safety in the COVID-19 era (PPE-SAFE): An international survey", Journal of Critical Care, 2020, vol. 59, pp.70-75. 